Supporting Information for

Probing the chiral domains and excitonic states in individual $\mathrm{WS}_{\mathbf{2}}$ tubes by second harmonic generation

Heming Xia, ${ }^{1}$ Xinyu Chen, ${ }^{1}$ Song Luo, ${ }^{1}$ Feng Qin, ${ }^{2}$ Alexander Idelevich, ${ }^{3}$

Saptarshi Ghosh, ${ }^{3}$ Toshiya Ideue, ${ }^{4}$ Yoshihiro Iwasa, ${ }^{4}$ Alla Zak, ${ }^{3}$ Reshef

Tenne, ${ }^{5}$ Zhanghai Chen, ${ }^{6}$ Wei-Tao Liu, ${ }^{1 *}$, Shiwei Wu ${ }^{1,7,8,9^{*}}$

1 State Key Laboratory of Surface Physics, Key Laboratory of Micro and Nano Photonic Structures (MOE), and Department of physics, Fudan University, Shanghai 200433, China

${ }^{2}$ College of Engineering and Applied Sciences, Nanjing University, Nanjing 210093, China

${ }^{3}$ Faculty of Sciences, Holon Institute of Technology, 52 Golomb Street, P.O. Box 305, Holon 5810201, Israel

${ }^{4}$ Quantum-Phase Electronics Center (QPEC) and Department of Applied Physics, The University of Tokyo, Tokyo 113-8656, Japan

${ }^{5}$ Department of Molecular Chemistry and Materials Science, Weizmann Institute of Science, Rehovot 76100, Israel

${ }^{6}$ Department of Physics, College of Physical Science and Technology, Xiamen University, Xiamen 361005, China

${ }^{7}$ Shanghai Qi Zhi Institute, Shanghai, 200232, China

8 Institute for Nanoelectronic Devices and Quantum Computing, Fudan University, Shanghai 200433, China

${ }^{9}$ Shanghai Research Center for Quantum Sciences, Shanghai 201315, China

*Corresponding email: swwu@fudan.edu.cn, wtliu@fudan.edu.cn

The supplementary information includes:

Supplementary Text 1-2.

Supplementary Figure S1-S3.

Supplementary Table S1. 


\section{Supplementary Text}

\section{The validity of the electric dipole approximation.}

Even in the case of normal incidence, high NA of a microscope objective may cause an effective oblique incidence and induce higher-order multipoles and magnetic components beyond electric dipole approximation. To rule out such possibility, we illuminated a single nanotube by three objectives with different NA. The results are shown in Figure S2. Co-polarized and crosspolarized SHG patterns are nearly coincident even by a microscope objective with low NA. Therefore, our results can be well described in the electric dipole approximation.

\section{Simulation of SHG polarization patterns with a bond additivity model.}

To analyze the SHG responses from multi-walled $\mathrm{WS}_{2}$ nanotubes with distorted honeycomb lattices, we use the bond additivity model (BAM) that has been successfully applied to two-dimensional (2D) honeycomb lattices under hydrostatic pressure. ${ }^{1}$ The BAM provides an analytical relationship between the macroscopic second-order nonlinear optical susceptibility tensor $\overleftrightarrow{\chi}^{(2)}$ and microscopic quantities, such as the bond length and bonding angle. ${ }^{2}$ We start from a 2D lattice with the 3-fold rotational symmetry shown in Figure S3a, whose basic structural unit includes three linear bonds sitting in its molecular coordinates $(\xi, \eta, \zeta)$ and can be represented by an equilateral triangle shown in Figure S3b. The optical response could be regarded as the superposition of that from three identical transition dipole moments. Since hyperpolarizability tensor $\stackrel{\leftrightarrow}{\beta}^{(2)}$ of each bond is the direct product of the transition dipole moment and Raman tensor, ${ }^{3}$ we denote the transition amplitude of each bond to be $\mu_{0} \zeta$, where $\zeta$ is along the direction of the dipole moment, and consider only the Raman tensor element $R_{\zeta \zeta}$ for this kind of linear bonds. ${ }^{4}$ So, the only nonzero hyperpolarizability tensor element of the bond is $\beta_{\zeta \zeta \zeta}^{(2)}$. With the hypothesis of 
all triangles forming the lattice being identical and aligned along the same direction, the macroscopic second-order nonlinear susceptibility $\overleftrightarrow{\chi}^{(2)}$ of the lattice equals $\mathrm{N} \overleftrightarrow{\beta}^{(2)}$, with $\mathrm{N}$ being the surface number density of the triangles and $\overleftrightarrow{\beta}^{(2)}$ being hyperpolarizability tensor of the equilateral triangular unit. By applying the Euler transformation to the three moments, we found the nonzero tensor elements for the 3-fold rotational symmetric system to be: $\left(\chi_{a a b}^{(2)}=\right)$ $a a b=a b a=b a a=-b b b$, with $a, b, c$ defined in Figure S3b. This agrees with that for a 3 -fold rotational symmetric, $2 \mathrm{D}$ system such as the $D_{3 h}$ point group. ${ }^{5}$

When the lattice deviates from the 3-fold rotational symmetry, at least one of the three moments must become inequivalent to the remaining. The difference can be in both the amplitude and the orientation of the transition dipole moment. We assume the $i$ th bond to tilt by an angle $\alpha_{i}$ away from its original direction, and the complex amplitude of transition dipole moment to change to $r_{i}=\mu_{0} \zeta \times \rho_{i} \mathrm{e}^{i \theta_{i}}$, where $\rho_{i}$ can be any positive value and $\theta_{i} \in[-\pi, \pi]$. These parameters are defined in Figure S3c. The hyperpolarizability tensor element then becomes $\beta_{\zeta \zeta^{\prime} \zeta^{\prime}}^{(2)}=\beta_{\zeta_{\zeta \zeta}^{(2)}} \times \rho_{i} \mathrm{e}^{i \theta_{i}}$. By repeating the procedures of coordination transformation, we find non-zero $\overleftrightarrow{\chi}^{(2)}$ elements for the distorted low symmetry system become:

$a a a, a a b=a b a=b a a, a b b=b a b=b b a, b b b$

with

$a a a \propto r_{1} \sin ^{3} \alpha_{1}+\cos ^{3} \frac{5 \pi}{6}+r_{2} \cos ^{3}\left(\alpha_{2}+\frac{\pi}{6}\right)$,

$a a b \propto r_{1} \sin ^{2} \alpha_{1} \cos \alpha_{1}+\cos ^{2} \frac{5 \pi}{6} \cos \frac{2 \pi}{3}+r_{2} \cos ^{2}\left(\alpha_{2}+\frac{\pi}{6}\right) \cos \left(\alpha_{2}+\frac{2 \pi}{3}\right)$,

$a b b \propto r_{1} \cos ^{2} \alpha_{1} \sin \alpha_{1}+\cos ^{2} \frac{2 \pi}{3} \cos \frac{5 \pi}{6}+r_{2} \cos ^{2}\left(\alpha_{2}+\frac{2 \pi}{3}\right) \cos \left(\alpha_{2}+\frac{\pi}{6}\right)$,

$b b b \propto r_{1} \cos ^{3} \alpha_{1}+\cos ^{3} \frac{2 \pi}{3} r_{2} \cos ^{3}\left(\alpha_{2}+\frac{2 \pi}{3}\right)$.

When we rotate the sample around its surface normal and detect the SHG response, the signal should depend on the projection of different tensor 
elements on the beam polarization planes. $\overleftrightarrow{\chi}^{(2)}$ elements expressed in the lab coordinates $(x, y, z)$ are related to those by the Euler transformation. In our experiments, we set the tube axis as y axis. By repeating the procedures of coordination transformation described above, we obtained the susceptibility tensor of the distorted lattice.

From above equations, we found the effective tensor elements responsible for our normal incident geometry are:

$\chi_{\|}^{(2)}(\phi) \propto\left[2 \cos \left(3\left(\phi-\phi_{0}\right)\right)-6 \cos \left(\phi-\phi_{0}-\frac{\pi}{3}\right)+2 \rho_{1} \exp \left(i \theta_{1}\right) \cos \left(3\left(\phi-\phi_{0}\right)-3 \alpha_{1}\right)+\right.$

$2 \rho_{2} \exp \left(i \theta_{2}\right) \cos \left(3\left(\phi-\phi_{0}\right)-3 \alpha_{2}\right)+6 \rho_{1} \exp \left(i \theta_{1}\right) \cos \left(\left(\phi-\phi_{0}\right)-\alpha_{1}\right)-$

$\left.3 \rho_{2} \exp \left(i \theta_{2}\right) \cos \left(\phi-\phi_{0}-\alpha_{2}\right)+3 \sqrt{2} \rho_{2} \exp \left(i \theta_{2}\right) \sin \left(\phi-\phi_{0}-\alpha_{2}\right)\right]$

and

$\chi_{\perp}^{(2)}(\phi) \propto\left[\rho_{2} \exp \left(i \theta_{2}\right) \sin \left(\phi-\phi_{0}-\alpha_{2}\right)-2 \cos \left(\phi-\phi_{0}+\frac{\pi}{6}\right)--2 \sin \left(3\left(\phi-\phi_{0}\right)\right)-\right.$

$2 \rho_{1} \exp \left(i \theta_{1}\right) \sin \left(3\left(\phi-\phi_{0}\right)-3 \alpha_{1}\right)-2 \rho_{2} \exp \left(i \theta_{2}\right) \sin \left(3\left(\phi-\phi_{0}\right)-3 \alpha_{2}\right)-$

$\left.2 \rho_{1} \exp \left(i \theta_{1}\right) \cos \left(\left(\phi-\phi_{0}\right)-\alpha_{1}\right)+3 \sqrt{2} \rho_{2} \exp \left(i \theta_{2}\right) \cos \left(\phi-\phi_{0}-\alpha_{2}\right)\right]$

Here, subscripts $\|$ and $\perp$ refer to the case with $\omega$ and $2 \omega$ beams polarized parallel and orthogonally, respectively (Figure S3d). $\phi$ refers to the angle between the polarization direction of $\omega$ beam and $y$ axis and $\phi_{0}$ refers to the angle between the original bond direction (armchair direction) and $y$ axis.

When the sample undergoes stress or bending, the lattice can be distorted, and the symmetry is lower. For a curved geometry like a nanotube, considering the large diameter of nanotubes $(\sim 100 \mathrm{~nm})$, each wall can still be regarded as a distorted honeycomb lattice approximately, whose basic structural unit can be expressed as Figure S3c. In other words, we can expect to model the structural unit of each wall as slightly distorted from the original equilateral triangle, and hence extract further geometric information about how the structural unit is distorted and arranged. 
We now apply the model to SHG experimental results from nanotubes as given in Figure 5. Compared with our experimental data shown in Figure S4ac, the simulation results are generally satisfactory in Figure S4 d-f. Note that fits of both $I_{\|}(\phi)$ and $I_{\perp}(\phi)$ at each position of nanotubes generated by the same set of parameters. The fitting parameters of bond additivity model are summarized in Table S1. As expected, from the change of $\rho_{i}$ and $\alpha_{i}$, we found that the lattice is distorted obviously. It is noted that as all our nanotubes are multiwalled, and the measured chirality is an average of multiple layers, thus, the values of $\phi_{0}$ indicate average angles between the original armchair direction and tube axis of this multiwalled nanotube. The directions of lobes of experimental co-polarized patterns are nearly as same as $\phi_{0}+n * 60^{\circ}$ ( $\mathrm{n}$ is an integer). Therefore, SHG polarization patterns allow us to unambiguously identify the chirality of individual $W_{2}$ nanotubes, which is defined by the orientation of native lattice relative to the tube axis. 


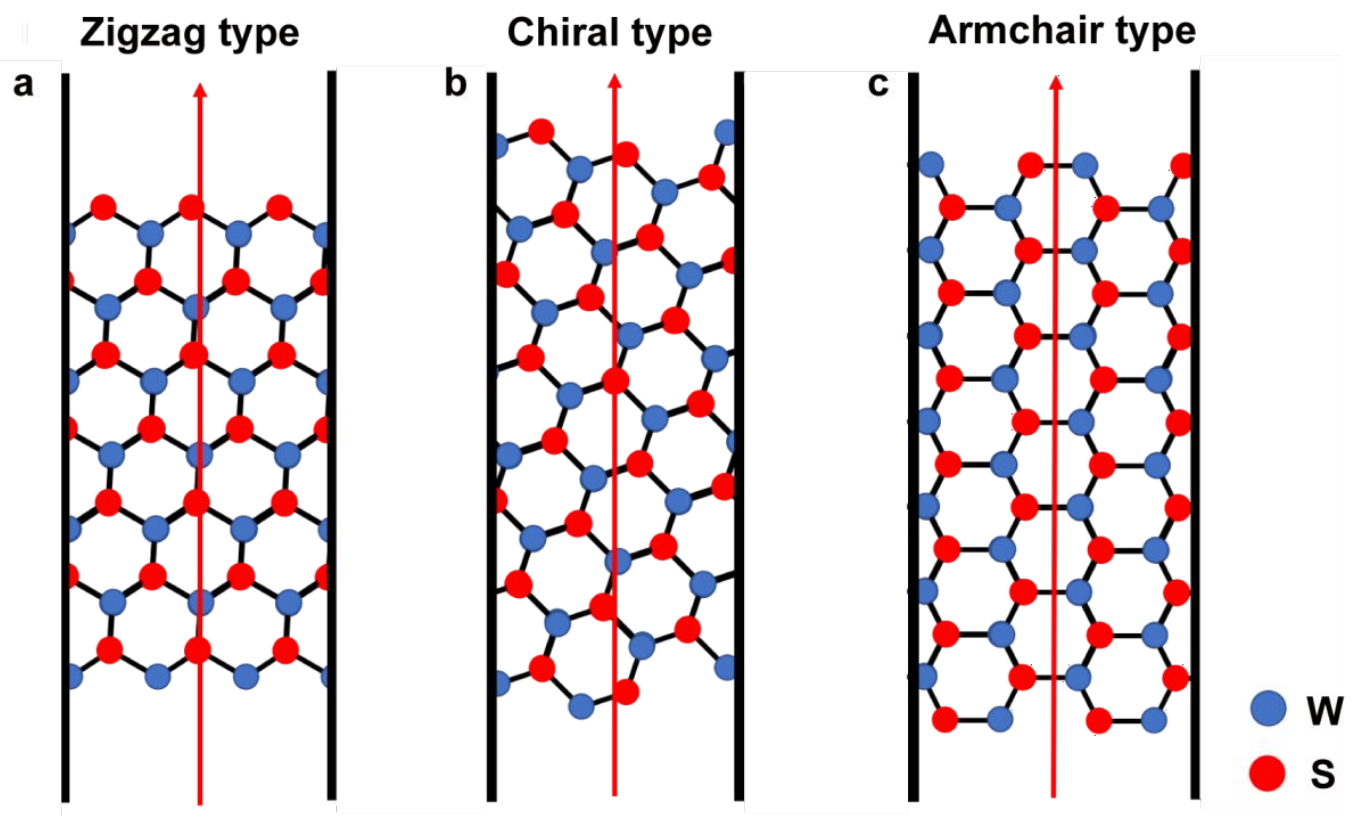

Figure S1. Illustration of the three typical structures of nanotubes. (a) zigzag type, (b) chiral type, and (c) armchair type. 


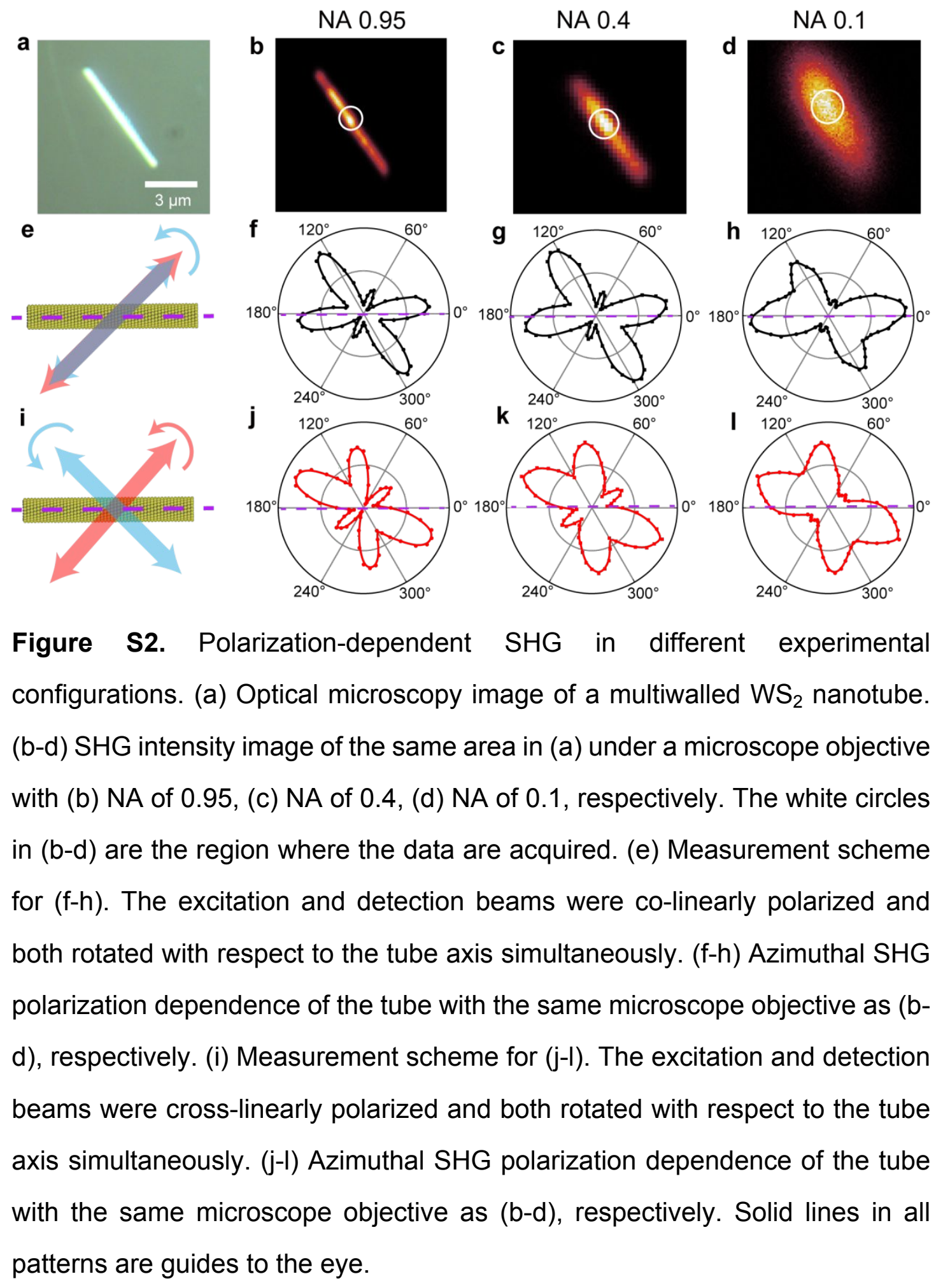




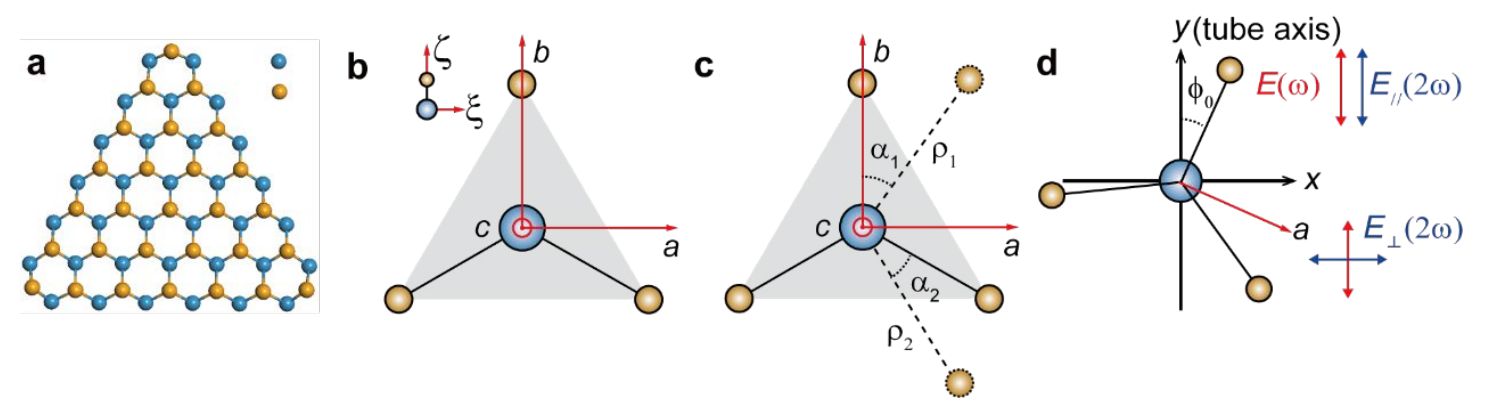

Figure S3. (a) Top view of the atomic structure of a hexagonal honeycomb monolayer; blue and yellow spheres represent different atomic species. (b) Definition of coordinates for a triangular structural unit. Inset shows the molecular coordinates for a single bond. (c) A distorted triangular structural unit with changes of two bonds. (d) Experimental geometry in lab coordinates. In our experiments, we set the tube axis as y axis. $\phi_{0}$ refers to the angle between the original armchair direction and $y$ axis. 


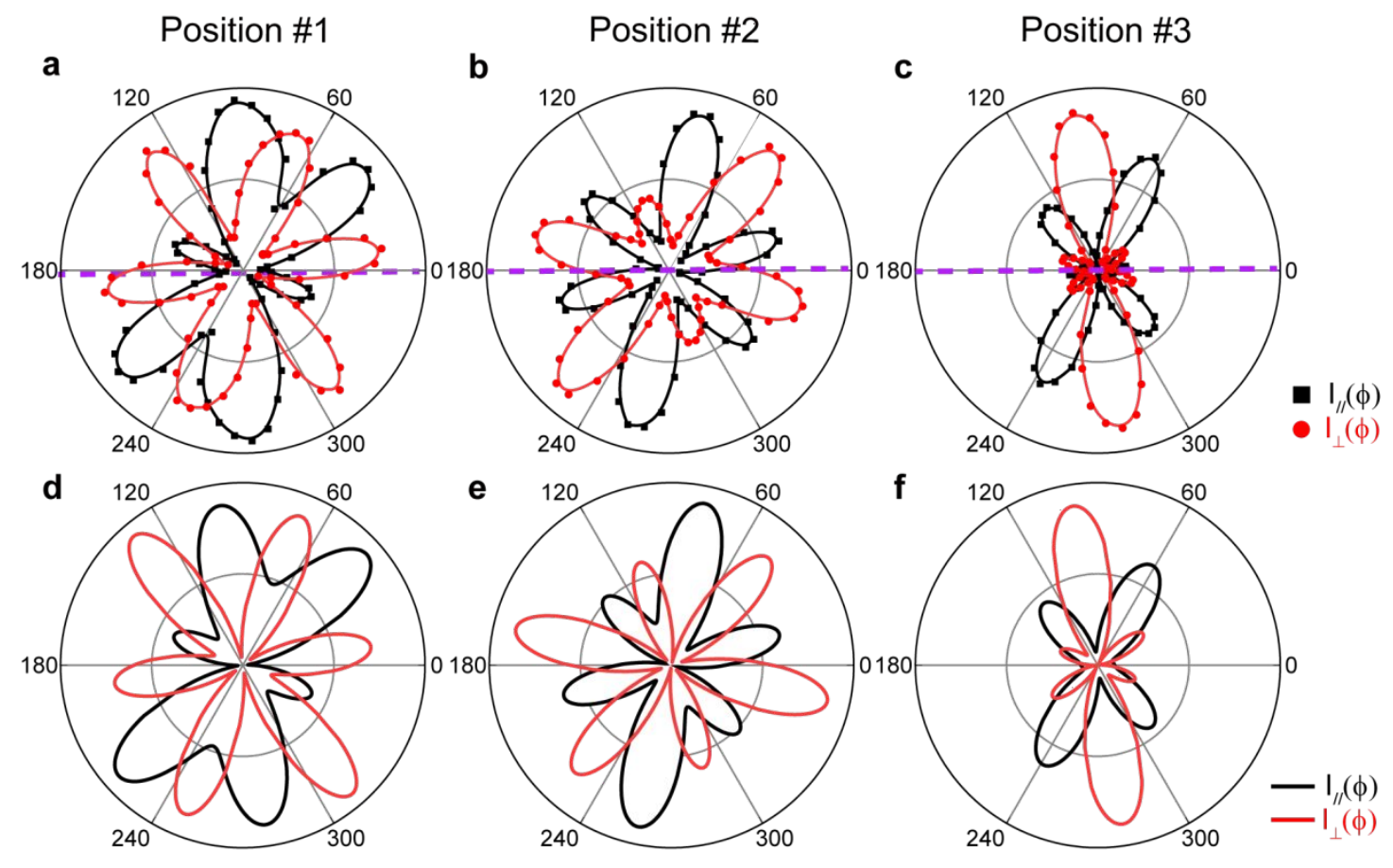

Figure S4. SHG anisotropy patterns and fitting results of three positions of the nanotube shown in Figure 1b. (a-c) Experimental data. Solid lines in patterns are guides to the eye. Dash lines (purple) are the direction of tube axis. (d-f) Theoretical simulations based on the bond additivity model. 


\begin{tabular}{|c|c|c|c|}
\hline Parameter & Position \#1 & Position \#2 & Position \#3 \\
\hline$\rho_{1}$ & $1.13 \pm 0.17$ & $1.00 \pm 0.37$ & $1.00 \pm 0.26$ \\
\hline$\theta_{1}\left(^{\circ}\right)$ & $\begin{array}{c}-58.49 \pm \\
3.34\end{array}$ & $-7.76 \pm 3.85$ & $\begin{array}{c}-83.66 \pm \\
9.20\end{array}$ \\
\hline$\alpha_{1}\left(^{\circ}\right)$ & $2.40 \pm 1.25$ & $-4.12 \pm 1.24$ & $-5.42 \pm 1.58$ \\
\hline$\rho_{2}$ & $0.89 \pm 0.16$ & $1.20 \pm 0.37$ & $1.05 \pm 0.24$ \\
\hline$\theta_{2}\left(^{\circ}\right)$ & $-48.21 \pm$ & $46.00 \pm 5.15$ & $-28.76 \pm$ \\
& 3.74 & & 8.65 \\
\hline$\alpha_{2}\left(^{\circ}\right)$ & $-3.01 \pm 2.00$ & $7.31 \pm 5.37$ & $-2.27 \pm 1.67$ \\
\hline$\phi_{0}\left(^{\circ}\right)$ & $36.56 \pm 1.12$ & $19.33 \pm 3.07$ & $8.98 \pm 3.18$ \\
\hline
\end{tabular}

Table S1. Parameters of bond additivity model applied to the three positions of nanotube shown in Figure 1b. The last row shows average angle between the original armchair direction and tube axis. 


\section{REFERENCES}

1. Lu, F.; Sun, Z.; Wu, S.; Liu, W.-T., Bond additivity model for anisotropic second-harmonic generation from two-dimensional honeycomb lattices. Opt. Lett. 2020, 45 (2), 268-271.

2. Hirose, C.; Akamatsu, N.; Domen, K., Formulas for the analysis of surface sum - frequency generation spectrum by $\mathrm{CH}$ stretching modes of methyl and methylene groups. J. Chem. Phys. 1992, 96 (2), 997-1004.

3. Wu, H.; Zhang, W.-k.; Gan, W.; Cui, Z.-f.; Wang, H.-f., Quantitative interpretation of polarization SFG vibrational spectra of air/methanol interface. Chin. J. Chem. Phys. 2006, 19 (3), 187-189.

4. Devine, T. M.; Adar, F., Raman spectroscopy of solids. Characterization of Materials 2002, 1-38.

5. Jiang, T.; Liu, H.; Huang, D.; Zhang, S.; Li, Y.; Gong, X.; Shen, Y. R.; Liu, W. T.; Wu, S., Valley and band structure engineering of folded $\mathrm{MoS}_{2}$ bilayers. Nat. Nanotechnol. 2014, 9 (10), 825-9. 